\title{
Lung Mass in a 28-Year-old Male: A Case Report of A RARE TUMOR
}

\author{
R. M. Mroz, M. Korniluk, E. Swidzinska, J. Dzieciol, J. Czaban, B. Panek, E. Chyczewska
}

Department of Lung Diseases and Tuberculosis, Department of Human Anatomy, Bialystok Medical University, Bialystok, Poland

\begin{abstract}
A twenty eight-year-old male presented with a two week history of dyspnea, cough, hemoptysis, chest pain, and fever $38-39^{\circ} \mathrm{C}$. He also complained of loss of appetite, general weakness and left leg pain for two months preceding admission. He was referred with suspicion of lung tumor to our institution. Chest Xray showed almost total atelectasis of the right lung with compensatory overinflation of the contralateral lung. Using computed tomography (CT), a lesion of diameter of $19.3 \times 14.1 \times 19.1 \mathrm{~cm}$ in the right lung, pleuritis, Th3 osteolysis, and compensatory overinflation of the left lung was seen. Bronchoscopy revealed a total obstruction of the right main bronchus due to submucosal infiltration and compression of the right main bronchus with negative histology of bronchial biopsy specimens. Transthoracic fine needle aspiration revealed celullae suspectae probabiliter neoplasmaticae suggesting tumor fusocellularis. USG of the abdomen revealed liver with numerous heterogeneous, solid areas hypo- and hyperechogenic, some of them with features of liquid or the disintegration up to diameter of $74 \mathrm{~mm}$. Subsequent fine needle aspirations of the thorax and liver revealed fibrolamellar hepatocarcinoma and carcinoma adenoides of the lung. Patient underwent chemotherapy with 5-FU/DDP/VCR with no response. This report presents a case of a rare lung metastasis from FL-HCC.
\end{abstract}

Key words: fibrolamellar hepatocarcinoma, carcinoma adenoides of the lung

\section{INTRODUCTION}

Fibrolamellar hepatocarcinoma is an unusual form of carcinoma occurring in young individuals [1]. It has distinct pathologic features and occurs typically without underlying hepatitis or cirrhotic liver and has prognosis slightly better than other forms of hepatocellular carcinoma (HCC) $[2,3]$. This report describes the clinical findings, radiologic evaluation, and histological features of such a case.

\section{CASE REPORT}

The study was performed according to the standards set by the Helsinki Declaration of 1975, regarding the Human Research and was approved by an institutional
Ethics Committee. Informed consent was obtained from the patient described in this article.

A twenty eight-year-old male presented with a two week history of dyspnea, cough, hemoptysis, chest pain, and fever $38-39^{\circ} \mathrm{C}$. He also complained of loss of appetite, general weakness and left leg pain for two months preceding admission. He was referred with suspicion of lung tumor to our institution. Chest Xray showed almost total atelectasis of the right lung with compensatory overinflation of the contralateral lung (Fig. 1A).

Using computed tomography (CT), a lesion of diameter of $19.3 \times 14.1 \times 19.1 \mathrm{~cm}$ in the right lung, pleuritis, Th3 osteolysis, and compensatory overinflation of the left lung was seen (Fig. 1B-D). Bronchoscopy revealed a total obstruction of the right main bronchus due to submucosal infiltration and compression of the right main bronchus with negative histology of bronchial biopsy specimens. USG of the abdomen revealed liver with numerous heterogeneous, solid areas hypo- and hyperechogenic, some of them with features of liquid or the disintegration up to diameter of $74 \mathrm{~mm}$ (Fig. 2).

Transthoracic fine needle aspiration (TFNA) revealed celullae suspectae probabiliter neoplasmaticae suggesting tumor fusocellularis (Fig. 3A). Subsequent TFNA revealed groups of carcinoma adenoides-like cells with some features of liver origin (Fig. 3B). Fine needle aspiration (FNA) of the liver revealed cancerous cells (Fig. 3C). Subsequent FNA of the liver revealed epithelial cells with nuclear atypia with characteristic pattern of nests and cords of malignant cells, which were separated by lamellar bands of dense, hypocellular collagen connective tissue, with round cytoplasmatic inserts characteristic for fibrolamellar hepatocarcinoma (Fig. 3D). Patient underwent chemotherapy with 5-fluorouracyl, cisplatin, and doxorubicin with no response.

\section{Discussion}

This patient was diagnosed with metastatic fibrolamellar carcinoma of the liver. Hepatocellular carcinoma is a highly malignant tumor, and the prognosis of HCC patients with extrahepatic metastases is poor [4], despite multimodality treatment applied [5]. Lungs are the most common localization of extrahepatic metastases of HCC. Fibrolamellar hepatocarcinoma (FL- 

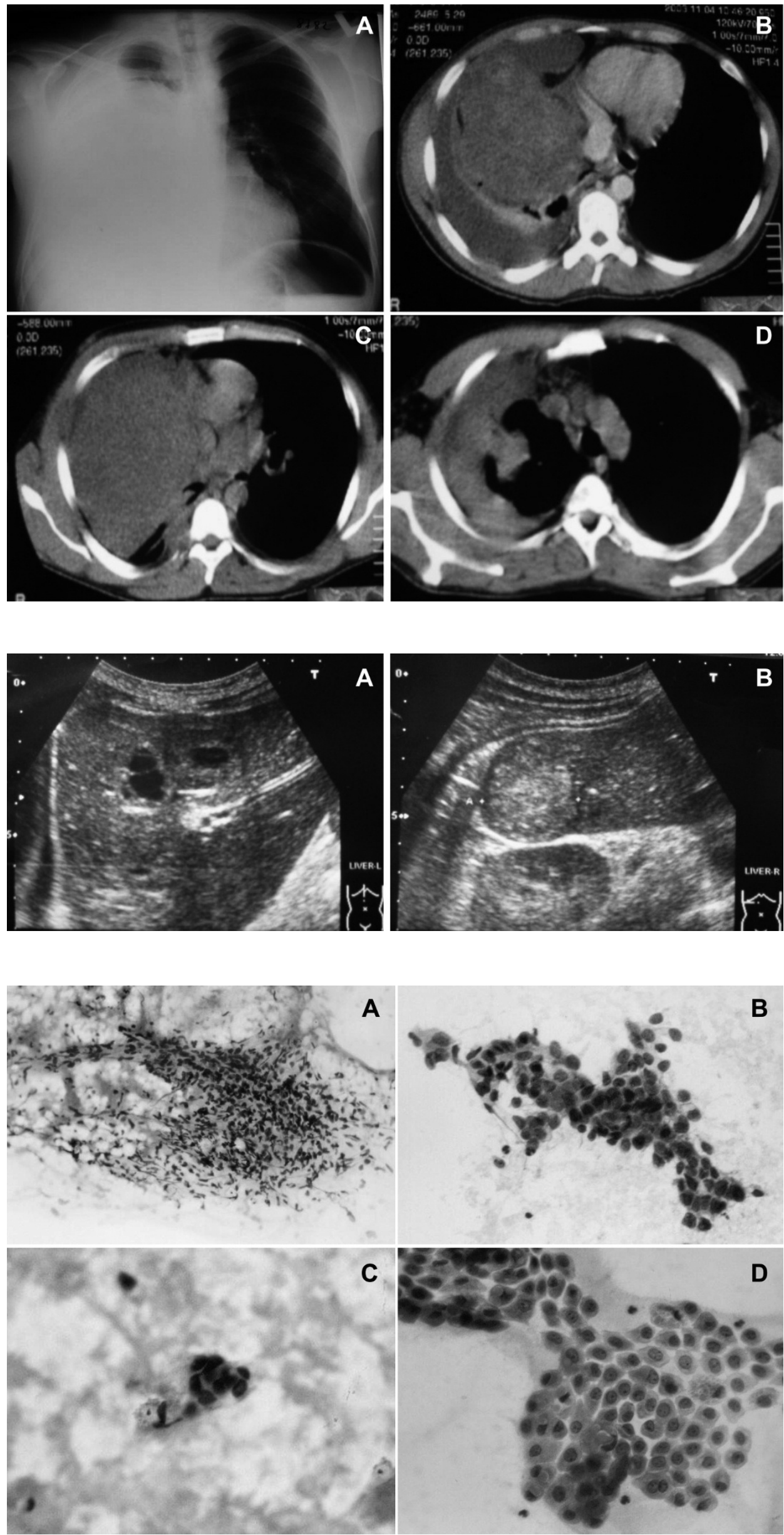

Fig.1. Chest X-ray: almost total atelectasis of the right lung with compensatory overinflation of the contralateral lung (A). CT: a lesion of diameter of $19.3 \times 14.1$ x $19.1 \mathrm{~cm}$ in the right lung, pleuritis, Th3 osteolysis, and compensatory overinflation of the left lung (B-D).

Fig. 2. USG of the abdomen. Liver with numerous heterogeneous (A), solid areas hypo- and hyperechogenic (B), some of them with features of liquid or the disintegration (A).

Fig. 3. Cytological findings. TFNA: celullae suspectae probabiliter neoplasmaticae (A), groups of carcinoma adenoides like cells with some features of liver origin (B). FNA of the liver: cancerous cells (C), epithelial cells with nuclear atypia with characteristic pattern of nests and cords of malignant cells, which were separated by lamellar bands of dense, hypocellular collagen connective tissue, with round cytoplasmatic inserts (D). 
HCC) was initially described by Edmondson in 1956. This rare variant of HCC accounts for less than 10\% of all cases of HCC, which number falls between 2$3 \%$ of all malignant cases in the United States [1]. FLHCC presents as a large, up to $20 \mathrm{~cm}$ diameter solitary intrahepatic mass with well-defined margins and a lobulated contour. In most cases regional lymph nodes are invaded at the time of diagnosis. Gross findings differentiate the FL-HCC from HCC as a well-demarcated, lobular, bile-stained, white or tan mass with a central stellate scar or fibrotic bands [7]. The defining feature of fibrolamellar carcinoma is its triad of histological characteristics, i.e., tumor cells with deeply eosinophilic cytoplasm, presence of macronucleoli and abundant fibrous stroma, arranged in thin parallel lamellae around the tumor cells [8]. FL-HCC cases are advanced in up to $80 \%$ of patients at the time of diagnosis, with regional adenopathy predominantly found in up to $70 \%$ of patients. Distant metastases occur in less than $20 \%$ of cases [7]. Lungs are most common sites of metastasizing HCC $[1,7]$, which is consistent with our case. In majority of HCC patients at the time of diagnosis distant metastases present as small solitary nodules. Uka et al [9] reported that mediastinal metastases occurred in $6.5 \%$ of 151 patients with extrahepatic metastases from HCC, and all of these were lymph node metastases. Ishii et al [5] reported pleural dissemination in 2 of 201 patients with extrahepatic metastases from HCC. However, many authors suggest more favorable prognosis of FL-HCC than that for conventional HCC [2, 9]. Kakar et al [8], following the World Health Organization blue book statements on fibrolamellar carcinoma, suggest refraining from any comment about its outcome compared to conventional hepatocellular carcinoma. According to their study fibrolamellar carcinoma is as aggressive tumor as HCC [8]. Tumor resectability is the basis of curative therapy for patients with HCC, but only approximately $30 \%$ of newly diagnosed patients undergo surgery and the 5 -year survival rate is less than $80 \%$ [1]. Moreover, most of the inoperable HCC cases remain unresponsive to chemotherapy, with very poor prognosis, and survival [10]. This was consistent with our observation. Pinna et al [11] reported that in pediatric patients who undergo liver resection frequencies of metastases to the liver, abdominal lymph nodes, lungs, and mediastinal lymph nodes were $44 \%, 33 \%, 29 \%$, and $10 \%$, respectively [11]. Prognostic factors such as multiple tumors, lymph node metastasis, and vascular invasion influence the survival time of FL-HCC cases [1]. These also are consistent with our findings. To date, there has been no report of a case presenting with huge lung mass as a consequence of lung metastases of fibrolamellar hepatocarcinoma. In conclusion, FLHCC is a rare histological variant of HCC and should be suspected in young patients with HCC without underlying liver disease. Despite relatively slow growth and late recurrence of HCC, FL-HCC should be suspected in case of rapid growth, advanced or multiple metastases of lungs, or peritoneum at the time of diagnosis.
Conflicts of interest: The authors had no conflicts of interest to declare in relation to this article.

\section{REFERENCES}

1. Liu S, Chan KW, Wang B, Qiao L. Fibrolamellar hepatocellular carcinoma. Am J Gastroenterol 2009; 104(10): 2617-24.

2. Wood WJ, Rawlings M, Evans H, Lim CN. Hepatocellular carcinoma: importance of histologic classification as a prognostic factor. Am J Surg May 1988; 155(5): 663-6.

3. Malouf G, Falissard B, Azoulay D, Callea F, Ferrell LD, Goodman ZD, Hayashi Y, Hsu HC, Hubscher SG, Kojiro M, Ng IO, Paterson AC, Reynes M, Zafrani ES, Emile JF. Is histological diagnosis of primary liver carcinomas with fibrous stroma reproducible among experts? J Clin Pathol 2009; 62(6): 519-24.

4. Natsuizaka M, Omura T, Akaike T, Kuwata Y, Yamazaki K, Sato T, Karino Y, Toyota J, Suga T, Asaka M. Clinical features of hepatocellular carcinoma with extrahepatic metastases. J Gastroenterol Hepatol 2005; 20: 1781-7.

5. Ishii H, Furuse J, Kinoshita T, Konishi M, Nakagohri T, Takahashi S, Gotohda N, Nakachi K, Yoshino M. Extrahepatic spread from hepatocellular carcinoma: who are candidates for aggressive anti-cancer treatment? Jpn J Clin Oncol 2004; 34: 733-9.

6. Edmondson HA. Differential diagnosis of tumors and tumor-like lesions of the liver in infancy and childhood. Am J Dis Child 1956; 91:168-86.

7. Craig JR, Peters RL, Edmondson HA, Omata M. Fibrolamellar carcinoma of the liver: a tumor of adolescents and young adults with distinctive clinico-pathologic features. Cancer 1980; 46: 372-9.

8. Kakar S, Burgart LJ, Batts KP, Garcia J, Jain D, Ferrell LD. Clinicopathologic features and survival in fibrolamellar carcinoma: comparison with conventional hepatocellular carcinoma with and without cirrhosis. Modern Pathol 2005; 18: 1417-23.

9. Uka K, Aikata H, Takaki S, Shirakawa H, Jeong SC, Yamashina K, Hiramatsu A, Kodama H, Takahashi S, Chayama K. Clinical feature and prognosis of patients with extrahepatic metastases from hepatocellular carcinoma. World J Gastroenterol 2007; 13: 414-20.

10. Okuda K. Natural history of hepatocellular carcinoma including fibrolamellar and hepato-cholangiocarcinoma variants. J Gastroenterol Hepatol 2002; 17: 401-5.

11. Pinna AD, Iwatsuki S, Lee RG, Todo S, Madariaga JR, Marsh JW, Casavilla A, Dvorchik I, Fung JJ, Starzl TE. Treatment of fibrolamellar hepatoma with subtotal hepatectomy or transplantation. Hepatology 1997; 26(4): 877 83.

Address for correspondence:

Robert M. Mroz

Department of Lung Diseases and Tuberculosis

Medical University of Bialystok

14, Zurawia St.

15-540 Bialystok

Poland

Phone: +48 857409530

Fax: +48 856545419

E-mail: robmroz@wp.pl 